\title{
Linx
}

Revue des linguistes de l'université Paris X Nanterre

38 | 1998

L'acquisition de la temporalité en situation bilingue

\section{La construction de la temporalité : étude basée sur l'observation d'un enfant bilingue}

The construction of temporality : case study of a French-Italian bilingual child

Silvia Romeo

\section{CpenEdition}

Journals

Édition électronique

URL : http://journals.openedition.org/linx/884

DOI : $10.4000 /$ linx. 884

ISSN : 2118-9692

Éditeur

Presses universitaires de Paris Nanterre

Édition imprimée

Date de publication : 1 décembre 1998

Pagination : 115-144

ISSN : 0246-8743

\section{Référence électronique}

Silvia Romeo, «La construction de la temporalité : étude basée sur l'observation d'un enfant bilingue », Linx [En ligne], 38 | 1998, mis en ligne le 28 juin 2012, consulté le 02 mai 2019. URL : http:// journals.openedition.org/linx/884; DOI : 10.4000/linx.884

Ce document a été généré automatiquement le 2 mai 2019.

Département de Sciences du langage, Université Paris Ouest 


\title{
La construction de la temporalité : étude basée sur l'observation d'un enfant bilingue
}

The construction of temporality : case study of a French-Italian bilingual child

\author{
Silvia Romeo
}

\section{Introduction}

1 Cet article porte sur l'acquisition du système de la temporalité chez l'enfant, vue comme appropriation graduelle des catégories conceptuelles sous-jacentes aux manifestations linguistiques et inférables grâce à celles-ci. La perspective développementale de notre approche, en amont de l'analyse présentée ici, tient compte des modifications, i.e. des complexifications des formes et des fonctions qui se restructurent les unes par rapport aux autres.

2 Les productions linguistiques analysées appartiennent à un moment où la capacité linguistique de l'enfant est déjà relativement développée, c'est-à-dire quand la grammaticalisation des relations temporelles est mise en place. La participation de deux langues au développement cognitif rend le dialogue entre représentation conceptuelle et représentation linguistique plus complexe. Mais en même temps, la disponibilité d'un double système de signes nous aide à comprendre un peu mieux la perception enfantine du concept de temps et la formation d'un système temporel.

3 Les théories de Piaget concernant les étapes du développement cognitif des enfants entre 2 et 7 ans ont constitué la base d'une grande part des recherches tentant de relier l'acquisition du langage à la cognition. En particulier pour ce qui concerne la structure temporelle, l'interprétation standard de la théorie de Piaget a été que la construction de la séquence temporelle est établie sur le principe de la réversibilité temporelle, et qu'elle 
ne fait donc pas partie des capacités cognitives enfantines avant le stade des opérations concrètes, à l'âge de 7 ans environ (French $1986: 128$ ).

4 La vaste littérature sur la question du développement de la temporalité (notamment Antinucci et Miller 1976, Stephany 1986) repose pour la plupart sur le postulat piagétien que les enfants jusqu'à 6-7 ans ne peuvent pas placer les événements dans le temps, et donc que la morphologie verbale précoce ne joue pas sa fonction déictique. En revanche, ils seraient capables de concevoir et de coder des oppositions aspectuelles entre situations. Ces chercheurs ont supposé que les enfants font l'équivalent des distinctions entre accompli / inaccompli, perfectif / imperfectif, qu'ils expriment par la morphologie temporelle passé / non passé.

5 Les théories de Piaget en question ont fait l'objet de lourdes critiques, surtout pour ce qui concerne l'âge auquel certaines habiletés cognitives sont initialement manifestées. En même temps, l'hypothèse décrite ci-dessus et que Weist $(1986,1989)$ a appelé l'Hypothèse $d u$ Temps Défectif a été aussi mise en question. Weist a formulé une théorie des systèmes temporels de la langue enfantine fondée sur les travaux théoriques de Reichenbach, Comrie (1985) et Smith qui vise à rendre compte de l'évolution du système référentiel temporel. Il a structuré en quatre étapes cette progression, du système initial « hic et nunc ", jusqu'au niveau le plus complexe de l'expression temporelle :

1. ST (Speech Time; en français temps de la parole TP) : le langage de l'enfant est limité à son environnement perceptif immédiat présent / absent ; la morphologie verbale n'est pas mobilisable pour des distinctions temporelles.

2. ET (Event Time; en français temps de la situation TS) : l'enfant commence à exprimer des relations déictiques entre TP et TS : il distingue les événements antérieurs, simultanés et postérieurs à TP. Les différences sont codées par un contraste passé vs non passé. Le temps de référence reste au TP.

3. RT (Reference Time; en français temps de référence TR) défini comme le contexte temporel pour un événement.

3a) $\mathrm{RTr}$ (restricted ; en français Temps de Référence restreint TRr) : le TR commence à se différencier du TP, mais le TR soit incorpore le temps de l'événement, soit reste au TP.

6 Etape suivante et ultime 3b) : RTf (free Reference Time ; en français temps de la Référence libre TRl ) : l'enfant peut manipuler les concepts de TP, TS et TR librement.

7 Dans cette séquence de développement, chaque système contient un réseau de concepts temporels inter-reliés. L'argument fondamental de Weist (1989) est que les innovations dans l'évolution des systèmes temporels ont des implications indirectes quant à la nature du schème conceptuel. En particulier, il souligne que l'émergence de relations temporelles dans la langue de l'enfant est une condition suffisante pour inférer des changements dans l'organisation conceptuelle.

Les données linguistiques que Weist a analysées d'enfants polonais lui ont permis de montrer l'acquisition précoce de la morphologie verbale et des moyens linguistiques de la temporalité, et de conclure que ces enfants avaient acquis les notions temporelles sousjacentes. Il a pu ainsi avancer à l'âge de 3 ans la période charnière de l'appropriation des 
concepts temporels de TP, TS, TR et la coordination des trois intervalles temporels, et remettre en cause l'âge " critique » postulé par les travaux antérieurs.

Weist a proposé une série hypothétique de stades de développement cognitif fondés sur une interaction étroite entre développement linguistique et développement cognitif :

- Système TP - stade de la permanence de l'objet. En s'appuyant sur diverses expériences en psychologie cognitive, Weist souligne que les enfants avant un an arrivent à construire des représentations d'objets et d'événements, donc à conceptualiser une relation temporelle primitive (TS(it) AV TP), à une phase de leur développement linguistique où les relations déictiques entre TP et TS ne sont pas encore codées.

- Système TS - stade du déplacement, la capacité d'utiliser l'information stockée dans la mémoire à long terme, qui demande que l'enfant comprenne la relation antérieurpostérieur.

- Systéme TRr - stade de la perspective temporelle non égocentrique - décentration : la capacité de prendre comme point de référence un autre que soi-même.

- Système TRl - stade de la mise en série et de la réversibilité : la capacité de contrôler les mécanismes d'ordre pour placer les notions de TP, TS, TR à des intervalles distincts dans le temps.

En s'appuyant sur l'hypothèse de Weist, on analysera ${ }^{1}$ le développement des systèmes temporels d'un enfant bilingue italo-français. On tentera de montrer comment à partir de deux ans et demi, cet enfant est entré dans la phase du temps de référence, et comment trois mois après, il a accédé au système du TR libre.

11 On traitera successivement les points suivants: exposition des méthodes d'analyse adoptées; description du système temporel de l'italien; analyse de productions significatives aptes à valider ou infirmer l'hypothèse de Weist ; comparaison avec d'autres données. Enfin, dans la discussion finale, on avancera quelques hypothèses sollicitées par nos résultats.

\section{Méthodes de collecte, de décryptage et d'analyse des données}

12 Cette contribution s'inscrit dans le cadre d'une recherche longitudinale en cours qui vise à étudier la construction de la référence aux procès et de leurs placements dans le temps chez un enfant bilingue à travers ses deux langues.

On a exploité une partie d'un vaste corpus enregistré et filmé, de l'âge de 24 mois jusqu'à 6 ans $^{2}$, à quoi s'ajoutent des observations notées au fil des jours. La possibilité d'accès direct et continu au monde construit jour après jour par l'enfant et notre participation, en tant que parent, à la réalisation de son "savoir " ont permis une interprétation contextuelle des énoncés de l'enfant et un aperçu de ses «théories naïves sur le monde qui sont supposées elles-mêmes guider l'acquisition des concepts» (Cordier 1994). On a pu mesurer l'avantage d'étudier un enfant bilingue pour la possibilité de valider la compréhension de son lecte, soit à travers la prise en compte des manifestations comparables dans les deux langues, soit à travers la traduction immédiatement après une ambiguïté. Une exploration contrastée des productions dans les deux langues a été menée à partir de la même grille d'analyse conceptuelle. Notre démarche part de l'analyse linguistique pour effectuer des inférences sur le développement conceptuel de l'enfant. Aucune analyse quantitative ne sera proposée dans cet article: comme Vygotsky 
(1934/85), nous croyons à l'intérêt d'une analyse qualitative qui cherche à révéler les relations causales ou dynamiques, d'une analyse développementale qui retourne à la source et reconstruit tous les points dans le développement d'une structure donnée.

14 Les choix méthodologiques adoptés ici se fondent sur une approche conceptuelle des structures temporelles, à partir d'un appareil conceptuel initialement conçu et éprouvé dans le programme de recherche sur l'acquisition de langues étrangères par des adultes de la Fondation Européenne de la Science ${ }^{3}$. La conceptualisation du modèle est due à Klein (1988) : c'est un modèle intégré de la temporalité (relations temporelles, aspect et mode d'action) reposant sur les relations entre différents types d'intervalles temporels. Nous présentons schématiquement dans le tableau A l'organisation des notions de base pour les trois grands types de temporalité, sous l'éclairage de Noyau $(1990,1991)$ : relations temporelles, perspective temporelle, temporalité inhérente des procès (pour un traitement détaillé, cf. Noyau 1991, chap. 2, dont nous reprenons les éléments de ce tableau).

Tableau A

\begin{tabular}{|l|l|}
\hline Intervalles temporels & (chez Reichenbach) \\
\hline a) MP moment de la parole & S speech time \\
\hline MSit moment de la situation & E event time \\
\hline MQ moment en question & R reference time \\
\hline Relations temporelles & \\
\hline a) relations d'ordre & \\
\hline Avant (AV) & \\
\hline Après (APR) & \\
\hline b) relations topologiques & \\
\hline concomitance & $( \pm$ CONT) \\
\hline adjacence & $( \pm$ ADJ) \\
\hline proximité & $( \pm$ PROX) \\
\hline Perspectives temporelles & \\
\hline - imperfectif & \\
\hline - perfectif & \\
\hline - accompli & \\
\hline Temporalité interne: propriétés temporelles inhérentes des procès : & \\
\hline
\end{tabular}




\begin{tabular}{|l|l|}
\hline - clôture des procès \pm CLO G (gauche) / \pm CLO DR (droite) & \\
\hline - durée ( \pm DUR) & \\
\hline - changement $( \pm$ CH) & \\
\hline - transition entre états ( \pm DIST) & \\
\hline
\end{tabular}

\section{Les données}

\subsection{Description du système temporel de la langue italienne}

15 Au niveau formel ${ }^{4}$ :les Temps italiens déploient un haut degré de synthéticité : ils sont codifiés à travers la suffixation, le déplacement de l'accent, l'alternance du radical. Les Temps sont exprimés par la flexion verbale (pour les Temps simples) et par la flexion verbale combinée à la syntaxe du verbe (Temps composés, périphrases, auxiliarisations). La flexion du verbe exprime un système de catégories grammaticales assez riche et amalgame les catégories de temps, mode, aspect et les catégories de personne et de nombre. Les oppositions phonologiques transparentes de la langue orale facilitent une saisie de la morphologie verbale (cf. Giacalone Ramat 1993 b).

\section{Au niveau fonctionnel (sémantico-conceptuel) ${ }^{5}$ :}

Les fonctions aspectuelles sont liées au Temps ou exprimées par des constructions périphrastiques mais "L'Aspetto non è quasi mai una categoria rigorosamente manifestata sul piano morfologico. In molti casi, la valenza aspettuale deve essere individuata per inferenza (...)».

«L'Azione e una categoria dai contorni (...) sfumati. Accanto a verbi che manifestano una data azione come proprio attributo inalienabile, ve ne sono altri che subiscono in maniera determinante l'influsso del contesto; e ve ne sono addirittura alcuni che mutano la proprio valenza in rapporto al singolo Tempo al quale sono coniugati, per effetto del diverso Aspetto che lo contraddistingue " (Bertinetto, 1986 : 99).

\subsubsection{Passato Remoto versus Passato Prossimo ${ }^{6}$}

17 L'italien est une langue à forte variation régionale. Sans considérer ici les dialectes, pour le sort du PRe dans la langue orale on remarque :

- en Italie du Nord absence du PRe, remplacé par le PPx

- en Italie du Centre, opposition entrePRet et PPx

- en Italie du Sud, absence du PPx, remplacé par le PRe

18 En italien standard, normalement parlé en Toscane (l'input familial de notre sujet), le PRe est encore d'usage fréquent et il a conservé, sur le plan sémantico-conceptuel, les traits suivants (cf. Bertinetto) :

- achèvement nécessaire du procès AV MP ;

- déicticité de la désignation temporelle (référée au passé) ;

- référence temporelle spécifique, pas d'interprétation éventuelle ou habituelle, ou détemporalisée. 
L'union des deux traits de perfectivité et de déicticité, toujours inhérents au PRe, entraine une implication pragmatique d'achèvement. Donc, dans un contexte bien défini, son emploi est obligatoire. Le PRe "forma aspettualemente pura» a un sens dynamiqueingressif et/ou aoristique. Il signale que le plan temporel sur lequel les événements sont disposés est nettement décroché de la situation existentielle du locuteur.

En opposition avec le PRe, le PPx possède les traits :

- fonction aspectuelle d'accompli et pertinence du procès ou de ses conséquences jusqu'au MP (« perfect » cf. Comrie 1976);

- référence temporelle qui n'est pas nécessairement déictique, simple antériorité par rapport au MQ qui peut se trouver au passé ou au futur, également désignation chronologique vide comme dans les usages atemporels ;

- achèvement indéterminé du procès, même dans l'acception «inclusive» qui est de sens imperfectif.

21 Au plan formel, le morphème du PRe est perceptivement saillant et phonétiquement stable, sauf exception, tandis que le PPx présente une morphologie stable mais non saillante.

On a évoqué le cas du PRe ici pour plusieurs raisons :

- La plupart des études sur l'acquisition de l'Italien (L1 ou L2) sont fondées sur la variété du Nord, où le PPx a désormais remplacé le PRe, au moins pour la langue orale.

- La présence du PRe dans nos données en italien nous permet de contraster les relations et les perspectives temporelles issues de ce Temps en italien avec celles liées au PC en français.

En nous appuyant sur Bertinetto, nous proposons dans le schéma ci-dessous les fonctions, i.e. les traits sémantico-conceptuels possibles pour chaque Temps verbal - sans spécifier les aires d'usage. Nous mettons en regard les termes du projet ESF présentés au tableau A et les termes employés par Bertinetto.

Tableau B

Presente, Imperfetto, Futuro Semplice, Trapassato Prossimo

PR (canto) :

\begin{tabular}{|l|}
\hline Propriétés temporelles \\
\hline $\begin{array}{l}\text { déictiques (MSit CON MQ-MP, MSit AV MQ-MP, MSit APR MQ-MP (Profutur)); non déictiques } \\
\text { (atemporel, omnitemporel) }\end{array}$ \\
\hline Propriétés aspectuelles \\
\hline imperfectives ( habituel, progressif, narratif, prophétique) \\
\hline perfectives (ingressif, dramatique, reportif) \\
\hline $\begin{array}{l}\text { PR indique (+ADJ) MP, et son aspect est imperfectif, en l'absence d'indications contraires du } \\
\text { contexte. }\end{array}$
\end{tabular}


IMP (cantavo) :

Propriétés temporelles

(MSit AV MP et MQ IN MSit) Simultanéité dans le passé, Présent dans le passé, Futur dans le passé

Propriétés aspectuelles

imperfective (continu, progressif, habituel)

perfective (narratif)

neutre

Propriétés modales

onirique, ludique, atténuatif, potentiel, hypothétique

FUT simple (canterò) :

\section{Propriétés temporelles}

déictique (MSit APR MQ-MP)

non déictique (omnitemporel)

Propriétés aspectuelles

perfective

sens secondaires imperfectifs : habituel, progressif, continu

Propriétés modales : épistémique

PQP (avevo cantato) :

Propriétés temporelles

non déictique (MSit AV MQ AV MP)

Propriétés aspectuelles

perfective (accompli)

imperfective (habituel)

Propriétés modales 
hypothétique, atténuatif, diminutif

\subsubsection{Langue italienne vs langue française}

temporels italien et français sont les suivantes :

- au niveau morphologique: la transparence de l'italien vs l'opacité du français; l'italien forme davantage les Temps de manière synthétique alors que le français tend à employer plus de constructions analytiques, surtout dans la langue orale ;

- au niveau fonctionnel : dans la langue orale, l'italien utilise un plus grand nombre de Temps Verbaux que le français. La morphologie verbale italienne est plus spécialisée que la française, et elle encode les fonctions de manière plus univoque. On peut poser que la restriction des possibilités d'interprétation ${ }^{7}$ entraîne une ambiguïté moindre du discours en italien comparé au français.

\subsection{Le sujet}

Jusqu'à l'âge de $2 ; 6, \mathrm{G}$ a été exposé surtout à la langue italienne (langue de la mère) et dans une moindre mesure à la langue française (langue du père). Il a vécu principalement en France, avec peu de contacts à l'extérieur de la famille, mais il a séjourné longuement en Italie où il a pu profiter de contacts interpersonnels riches et variés. Depuis l'âge de $2 ; 6$, il a passé une partie de la journée dans une crèche familiale francophone, où il a diversifié un peu son environnement linguistique. Les conditions de vie jusqu'à l'âge de trois ans, compte tenu des situations (la mère qui a contrebalancé l'environnement francophone), lui ont permis un développement assez équilibré des deux langues. Le mélange des langues est très rare. Jusqu'à $2 ; 2$, il avait tendance à commencer son discours dans une langue, et ensuite, grâce au feedback reçu de son interlocuteur, il changeait de code en fonction de la situation. La possibilité de s'exprimer dans les deux langues a contribué au développement de la compétence pragmatique : l'entraînement à soutenir une conversation avec deux interlocuteurs de deux langues en même temps a influé sur sa capacité à « traduire » ses acquis d'une langue à l'autre.

\subsection{Synthèse du développement des formes selon le système de Weist}

On propose un schéma provisoire du développement de la morphologie verbale, des adverbes, des connecteurs et des subordonnées en les rapportant aux phases progressives envisagées par Weist. La tranche d'âge indique la période à laquelle une forme est intéressée par le phénomène du 'coup de projecteur' (cf. Slobin (1985) «spotlight effect »).

Avec le développement cognitif, les formes de base acquièrent une spécification fonctionnelle prototypique, lors d'une étape particulière de concentration («spotlight ») cognitive et linguistique, soulignée par un emploi massif de ces formes et fonctions. Dans le développement de chaque forme, l'unifonctionnalité acquise laisse ensuite la place à une capacité de plus en plus variée d'emploi des formes (plurifonctionnalité, cf. 6.1 infra). Ce processus concerne chacune des langues, mais le développement linguistique se 
faisant presque en parallèle, des renvois continus et des influences réciproques complexifient ultérieurement ce cadre évolutif. Dans le tableau $\mathrm{C}$, nous nous limitons à la liste des formes, la description des fonctions sémantiques assumées par les formes en contexte, qui sont développées surtout dans les deux premières phases, méritant un traitement à part ${ }^{8}$.

En ce qui concerne l'étiquetage par les signifiants en classes morphologiques des langues adultes de référence, des problèmes particuliers sont liés à la phase TP à cause des caractéristiques contextuelles de la langue enfantine à cette époque. On a essayé ici de présenter la phase TP avec la terminologie utilisée pour les phases suivantes à titre indicatif et par souci d'homogéneité. Les cas d'ambiguïté dans le discours de $G$ commencent a être plus rares à partir de deux ans. A la phase TS, très fertile pour l'acquisition des moyens linguistiques de la temporalité, le lecte de cet enfant se transforme rapidement jusqu'à devenir de plus en plus semblable à la langue adulte, à partir de $2 ; 3$, du point de vue formel, fonctionnel et pragmatique.

Tableau C

Phase : TP - Age : ( jusqu'à1 ;8)

\begin{tabular}{|l|l|}
\hline INF & INF et/ou PP \\
PP & PR et/ou IFT \\
PR et/ou IFT & encore \\
ancora (idée de répétition ou de supplément) & \\
\hline
\end{tabular}

Phase : TS - Age : $(1 ; 9-2 ; 4)$

\begin{tabular}{|c|c|}
\hline $\begin{array}{l}\text { PP (verbes téliques et atéliques) } \\
\text { PR }\end{array}$ & $\begin{array}{l}\text { PP (verbes téliques et atéliques) } \\
\text { PR } \\
\text { verbe modal vouloir } \\
\text { PC }\end{array}$ \\
\hline $\begin{array}{l}\text { PPX } \\
\text { GER } \\
\text { périphrase progressive } \\
\text { ("stare » au PR ou à l'IMP +GER) } \\
\text { «che » pronom relatif }\end{array}$ & $\begin{array}{l}\text { "c'est moi qui »+ phrase } \\
\text { déjà, puis, après }\end{array}$ \\
\hline $\begin{array}{l}\text { IMP } \\
\text { più/nonpiù,ora,poi,dopo,digià, oggi, domani, ieri } \\
\text { (indication d'heure) } \\
\text { perché se no + phrase } \\
\text { verbes modaux (fléchi+INF) }\end{array}$ & $\begin{array}{l}\text { IMP } \\
\text { (indication d'heure) } \\
\text { parce que sinon + phrase } \\
\text { verbes modaux } \\
\text { (fléchi+INF) }\end{array}$ \\
\hline
\end{tabular}




\begin{tabular}{|l|l|}
\hline COND & FP \\
périphrase (andare a +INF) & quand + phrase \\
quando + phrase & pour + phrase \\
per + phrase & encore ( = toujours), pas encore \\
ancora (persistance d'une action ou d'un état au MP) & $\begin{array}{l}\text { hier, demain, plus } \\
\text { tout de suite, maintenant, d'abord, ensuite, } \\
\text { FUT }\end{array}$ \\
& pas encore \\
\hline
\end{tabular}

Phase : TRr - Age : $(2 ; 5-2 ; 8)$

\begin{tabular}{|l|l|}
\hline $\begin{array}{l}\text { stare per }+\mathrm{INF} \\
\text { non ancora (ce qui doit se produire ne s'est pas pour le moment produit) } \\
\mathrm{PPR}\end{array}$ & $\begin{array}{l}\mathrm{PQP} \\
\text { phrase relative } \\
\text { phrase finale objective } \\
\text { en train de + INF }\end{array}$ \\
\hline $\begin{array}{l}\text { PRe } \\
\text { phrase relative et constructions }\end{array}$
\end{tabular}

Phase : TRI - Age : $(2,9-3 ; 0)$

\begin{tabular}{|l|l|}
\hline intanto, l'altro giorno, l'altro ieri & COND \\
SBJ Présent, Imparfait & FUT \\
se + phrase & SBJ Présent \\
fare +INF & si + phrase \\
forme passive & faire + INF \\
discours indirect & forme passive \\
& discours indirect \\
\hline
\end{tabular}

\section{Analyse des données ${ }^{9}$}

Dans ce qui suit, on se propose d'analyser :

- le développement des formes dans les phases TRr et TRl, en contrastant les différences fonctionnelles et pragmatiques dans les deux langues ;

- la capacité de construire des textes où la morphologie verbale est fonctionnelle pour structurer des événements situés dans le temps ;

- ce qui sous- entend, au niveau conceptuel :

- une saisie de la temporalité en termes de notions comme les relations d'ordre AV, APR, CONT, topologiques (ADJ, PROX), les perspectives temporelles (i.e phénomènes aspectuels), et les propriétés temporelles inhérentes des procès ; 
- les relations entre le niveau linguistique et le niveau conceptuel, en considérant le degré de complexité dans les productions en deux langues : jusqu'à quel point l'enfant est-il influencé dans sa sélection des faits du monde et dans leur expression en fonction des moyens linguistiques disponibles. Les caractéristiques intrinsèques des langues en question et du langage adressé à l'enfant entrent ici en jeu. On observe comment la transparence de la langue italienne peut orienter notre compréhension du lecte de l'enfant alors que l'opacité de la langue française nous oblige à inférer souvent le signifié.

L'exemple qui suit est extrait d'un jeu-test à partir d'images visant à faire exprimer par l'enfant le placement temporel d'un événement avant ou après un moment-repère fourni par une autre situation.

\begin{tabular}{|l|}
\hline Ex. 1 (G : 2 ;9)L'image représente un autobus et un garçon dans la rue avec ses valises. \\
\hline M. Allora il bimbo é sceso o deve ancora salire? \\
\hline G. Deve ancora salire \\
\hline M. Da cosa l'hai capito? \\
\hline G. guida ancora \\
\hline « 1 M. Alors le garçon est descendu ou doit encore monter? \\
\hline 2 G. Il doit encore monter \\
\hline 3 M. Qu'est ce qui te l'a fait comprendre? \\
\hline 4 G. (Le conducteur, indiqué du doigt) est encore en train de conduire »
\end{tabular}

31 Ce test met en évidence le stade de développement cognitif de la réversibilité. Mis devant le choix entre deux situations, MQ APR MSIT et MQ AV MSit, G opte pour la prospective, en justifiant son idée d'une manière conséquente. Il utilise deux fois l'adverbe de contraste encore pour marquer la persistance au MQ de l'action de conduire. Dans d'autres moments du même test, il utilise l'adverbe de contraste déjà pour la fonction inverse.

Dans les exemples qui suivent, antérieurs de quelques mois, on voit déjà la capacité d'exprimer la succession temporelle des procès, et le prélude au stade de la réversibilité.

\subsection{Phase $\mathrm{TRr}$}

Ex. 2 ( $G: 2 ; 5)$ ( Le jeu est déjà commencé : il y a des constructions et des personnages que $\mathrm{G}$ et la $\mathrm{M}$ ou le $\mathrm{P}$ font agir)

\begin{tabular}{|l|}
\hline a) G 1 Non ci sta \\
\hline 2 La levo \\
\hline 3 L'ho levata!
\end{tabular}




\begin{tabular}{|l|}
\hline 4 Casca! casca casca! \\
\hline 5 Ho aprito (pour “aperto") la porta \\
\hline « 1 Elle ne tient pas \\
\hline 2 Je l'enlève \\
\hline 3 Je l'ai enlevée! \\
\hline 4 Elle tombe, elle tombe, elle tombe \\
\hline 5 J'ai ouvert la porte " \\
\hline b) G 1 Elle va prendre les crèpes cette dame-là. \\
\hline 2 Prends les crèpes \\
\hline 3 Elle a mangé les crèpes \\
\hline
\end{tabular}

34 En 2 a), on remarque l'utilisation du PR pour décrire d'abord un état (1), puis (2) une action en cours MSit INCL MQ CON MP, ou bien une action à accomplir MSit APR +ADJ MP ; en (3), le PPx avec le même verbe que celui utilisé au PR pour une action accomplie MSit AV +ADJ MQ-MP.

35 (4) MSit CON MP ; (5.) comme (3). En 2 b), le même verbe prendre est utilisé d'abord au FP avec sa fonction d'action à venir MP AV +ADJ MSit, puis au PR sans sujet ou à la forme IFT, MSit CON MP. Le PC en (3) marque MSit AV +ADJ MQ-MP.

Dans ces ex. 2, on trouve une succession de procès décidés, accomplis et commentés lors de l'action par l'enfant maître du jeu, comme dans un reportage sportif ${ }^{10}$. On retrouve les mêmes fonctions dans l'exemple suivant qui a un contexte comparable : $G$ est spectateur des mouvements d'Eva et en parle à la $\mathrm{M}$ qui ne regarde pas.

\begin{tabular}{|l|}
\hline Ex. 3 (G. $2 ; 5)$ \\
\hline 1Guarda che sta facendo Eva! \\
\hline 2 Cosa sta facendo ora Eva! \\
\hline 3 Che faceva Eva alla tendina? \\
\hline " 1 Regarde ce qu'est en train de faire Eva! \\
\hline 2 Qu'est ce qu'elle est en train de faire Eva maintenant ! \\
\hline 3 Qu'est ce qu'elle faisait Eva au petit rideau? ? \\
\hline
\end{tabular}

Dans (1) et (2), il utilise une construction périphrastique pour marquer le progressif (MQMP IN MSit), souligné en 2 avec l'adverbe maintenant. En (3), quelques minutes après (2), il 
utilise le même verbe à l'IMP : la fonction imperfective est maintenue au fil des trois énoncés, ce qui change est la relation temporelle : AV MP en (3).

Ex. $4(G .2 ; 6)$

a) Qu' est ce qui s'est passé dans ma chambre?

b ) Che é successo nella mia camera... ? Che un giorno (le jour avant) papa et mamma hanno levato la poignée... Bisogna rimetterla.

b) «Qu'est ce qui s'est passé dans ma chambre? Qu'un jour papa et maman ont enlevé la poignée... Il faut la remettre.»

c) Il était une fois la Palucale qui faisait la dînette.

(Palucale, nom inventé par G. pour désigner le personnage matérialisé par un bourrelet de bas de porte)

Les ex. 4 semblent initier un discours narratif: on remarque les adverbes temporels de localisation indéterminée typiques des contes de fées il était une fois, un jour, pour référer à des événements passés qui ont eu lieu dans la chambre de G. Les différentes situations sont illustrées en tenant compte des propriétés inhérentes des procès : $+\mathrm{CH}$ dans $b$ ) exprimé avec un PPx en fonction perfective; +DUR dans c) avec un IMP en fonction imperfective. L'énoncé (b) prononcé tout de suite après a) est un exemple typique de traduction: G. reformule son énoncé du début avant de poursuivre dans le code demandé par la situation.

\begin{tabular}{|l|} 
Ex. 5 (G. 2 ;7) (M. et G. parlent d'un voyage prévu pour le lendemain) \\
\hline 1 M. Si parte domani \\
\hline 2 G. Ora è domani? \\
\hline «1 M. On part demain \\
\hline 2 G. Maintenant c'est demain?» \\
\hline
\end{tabular}

Le $\mathrm{n}^{\circ} 5$, très fréquent et typique de cet âge, montre le désir de $\mathrm{G}$. de modifier le temps par sa simple expression verbale. Avec un verbe être au PR qui permet d'opérer une identification entre les intervalles des deux adverbes, G. transforme MQ -PROX MP (exprimé par la mère ) en $\mathrm{MQ}+\mathrm{ADJ} \mathrm{MP}$, ce qui entraîne comme conséquence le déroulement de l'événement. On a donc l'opposition : (1) MP AV et -PROX MQ-MSit / (2) MP-MQ +ADJ MSit.

Dans l'ex. qui suit, le refus de faire, marqué par trois négations, redouble sa force avec une reformulation en italien qui est la traduction exacte de l'énoncé en français. 
Ex. $6(G ; 2 ; 7)$ (avant de partir chez la nourrice)

a) On va pas ni chez Laurence, ni chez Danielle, ni à la garderie

b) Non si va nè da Laurence, nè da Danielle, nè à la garderie

41 Dans l'ex. 7, $M$ et $\mathrm{G}$ construisent des espaces avec des pièces en bois brut sur lesquelles $M$ avait, antérieurement, dessiné des fleurs. Eva a 11 mois.

\begin{tabular}{|l|}
\hline Ex. 7 (G. 2 ;5) \\
\hline 1 M. Qui ci sono i fiori, ci puoi fare anche il giardino \\
\hline 2 G. Non li riconosce Eva \\
\hline 3 M. No, Eva non li conosce \\
\hline 4 G. Non l'aveva visto questo \\
\hline 5 M. L'aveva visto ma non se ne ricorda perché era piccolissima \\
\hline « 1 M. Ici il y a les fleurs, tu peux en faire un jardin, même \\
\hline 2 G. Eva ne les reconnaît pas \\
\hline 3 M. Non, Eva ne les connait pas \\
\hline 4 G. Elle ne l'avait pas vu celui-ci \\
\hline 5 M. Elle l'avait vu mais elle ne s'en rappelle pas parce qu'elle était toute petite." \\
\hline
\end{tabular}

42 L'affirmation de G. vise à clarifier la différence entre le verbe riconoscere et conoscere et à tester son propre raisonnement par inférence : «Si Eva ne connaît pas les fleurs (3) et non reconnaît (2) ça signifie qu'elle ne les avait pas vues avant. » Les énoncés (3) et (5) de la $\mathrm{M}$ essayent de faire comprendre la distinction entre identifier par la mémoire et former l'idée, le concept. G utilise le PQP avec une double fonction temporelle et aspectuelle : le $\mathrm{PQP}$ en (4) indique un MQ à valeur indéterminée situé antérieurement (-PROX : de 0 à 11 mois avant) au MSit exprimé en (2), à son tour antérieur (+PROX) au MP. La fonction du $\mathrm{PQP}$ donc est semblable à celle de l'adulte, la M en (5).

43 La sensibilité que montre $G$ pour les couples de mots aussi bien en italien qu'en français s'est manifestée très précocement et mérite un développement à part (cf. 6 infra). 


\subsection{Phase TRI}

44 Les exemples (8) à (18) illustrent des procédés linguistiques utilisés massivement à partir de $2 ; 9$. On peut noter que cet enfant se focalise sur plusieurs phénomènes linguistiques en même temps et au même âge (phrase avec quand, si, PRe, COND, SBJ, PQP).

\begin{tabular}{|l|}
\hline Ex. 8 (G. 2 ;9) (G. se trouve à côté de la fenêtre) \\
\hline M. I Bambini non devono stare vicino alla finestra \\
\hline G. Senno saltano dalla finestra... \\
\hline e poi entrano dalla porta \\
\hline e poi prendono l'ascensore \\
\hline e poi risalono (pour "risalgono") su? \\
\hline « M. Les enfants ne doivent pas rester a côté de la fenêtre \\
\hline G. Sinon ils sautent de la fenêtre... \\
\hline et puis ils rentrent par la porte \\
\hline et puis ils prennent l'ascenseur \\
\hline et puis ils remontent en haut? " \\
\hline
\end{tabular}

Mise en série temporelle d'actions et cyclicité. L'image ressemble beaucoup à la série d'actions pour jouer au toboggan et les verbes au PR suggèrent la répétitivité. Le choix du verbe saltare implique sur le plan sémantique un mouvement actif de la part des enfants.

Ex. $9(G .2 ; 9)$ à M

a) Domani (dans quelques heures) quando Papà ritorna, si monta il trenino

a) « Demain, quand Papa revient, on monte le train »

b) 1.Quando ho finito di lavorare

2. leggio ( au lieu de“leggo") i libri con te

b)« Quand j'ai fini de travailler je lis les livres avec toi »

c) E poi quando ho disegnato sui fogli li metto qua

c) «Et après quand j'ai dessiné sur les papiers je les mets ici » 
d) (la phrase est commencée) quando hai levato il divano

l'altro ieri.

d) « quand tu as enlevé le divan, avant hier "

e) (G;3 ) 1.Ti ricordi 2.quando ero al mare 3.e non avevo questo

(du sopalin) 4.e facevo questo? (geste de se nettoyer les mains en les tapant l'une contre l'autre).

e) «1. tu te rappelles 2. quand j'étais à la mer 3. et que je n'avais pas ça 4. et je faisais ça ?»

Dans les phrases a, b, c, les subordonnées sont introduites par le connecteur quando. Le PR de la phrase principale a la fonction de pro-futur.

47 Les phrases a) et d) ont un repérage temporel composite : anaphorique et déictique. Le PPx de la subordonnée accompagne en b) la clôture du procès (ho finito di lavorare), et en c) l'achèvement d'un procès +DUR (ho disegnato). En a), l'adverbe temporel demain ne correspond pas à la réalité temporelle de la situation, tandis que l'altro ieri de e) est utilisé à bon escient. En e), la localisation spatiale contribue au repèrage temporel. Le Temps IMP pour les deux verbes statifs (essere, avere) et le verbe d'action (fare) a une fonction imperfective.

Ex. 10 (G.2 ;9) (avant de sortir de la porte d'entrée)

Siamo partiti

«On est partis »

G. a transféré en italien l'expression que son père utilise en français quand il est pressé, pour dire qu'on sera bientôt parti, et il l'emploie avec la même fonction. La configuration temporelle est pragmatiquement MQ-MP CON MSiT et non pas MQ-MP APR MSiT.

\begin{tabular}{|l|}
\hline Ex. 11 \\
\hline a) (G. 3) 1.Perché hai messo la cassetta dentro? \\
\hline 2 M. Così \\
\hline 3 G. E si mette una pila dentro poi si pigia sul bottoncino poi la lampadina si accende? \\
\hline 4 M. Si \\
\hline 5 P. Perchè si è accesa la lampadina? \\
\hline 1 « G. Pourquoi tu as mis la cassette dedans? \\
\hline 2 M. Comme ça \\
\hline
\end{tabular}


$3 \mathrm{G}$. Et on met une pile dedans et puis on appuie sur le bouton et puis l'ampoule s'allume?

4 M. oui

5 P. Pourquoi s'est allumée l'ampoule?»

b) (G. 2 ;10) (Description de procès : G. fait à nouveau la série de gestes)

Stai attenta, fabbiamo messo questo qui poi abbiamo fatto passare questo sotto la porta, abbiamo fatto partire questo.

b) Sois attentive, nous avons mis cela ici puis nous avons fait passer ceci sous la porte nous avons fait partir celui- ci

c) (G. 3) (A la M.) (jeu de construction)

Questo viene bloccato qui e questo viene bloccato qui

c) « Celui-ci va être bloqué ici et ceci va être bloqué ici »

d) (G. 3.)... et après je mets un dedans et après.....un tournevis et

après on va faire bzz dans le mur et après on a fait le trou

dans le mur

Dans les ex. 11, on a une série de micro-actions enchaînées par après et poi, pour expliquer le fonctionnement d'un jeu. L'ordre est adéquat dans tous les exemples. Dans a), b), c), le temps est au PR, en c) le PR a la forme passive avec le verbe venire (qui peut remplacer formellement et sémantiquement l'auxiliaire essere). b) G. raconte et montre en même temps, en faisant à nouveau les mêmes gestes, une série d'actions qu'il vient d'accomplir. Il utilise le PPx avec sa fonction de +ADJ MP. En a), il y a alternance entre ancrages temporels - déictique au PPx en (1) et (5), et atemporel générique (3) au PR.

Dans le texte qui suit G. essaie de raconter le Chien Glouton de La Fontaine (G. 2 ;9,2). Le support est constitué seulement d'une image dans laquelle le chien, avec le beefsteak dans la gueule, regarde son reflet dans l'eau.

\begin{tabular}{|l|}
\hline Ex. 12 a) \\
\hline 1 M. Il cane golosone, dai \\
\hline 2 G. Voleva prendere la grande bistecca \\
\hline 3 M. Ce l'aveva già una in bocca, vero? \\
\hline 4 G. Il ruscello l'aveva presa \\
\hline 5 M. Si, aspetta.... \\
\hline
\end{tabular}




\begin{tabular}{|l|}
\hline 6 G. (continue sans écouter) Non gli bastava. \\
\hline 7 Prendeva ( =voulait prendre) anche questa bistecca (indique \\
\hline l'image) \\
\hline 8 M. Allora che fece? \\
\hline 9.G. Andò nell' acqua e 10. la prendò ( au lieu de"prese") \\
\hline " 1 M. Raconte le Chien glouton... \\
\hline 2 G. Il voulait prendre le grand beefsteak \\
\hline 3 M. Il en avait déjà un dans la bouche, n'est ce pas? \\
\hline 4 G. Le ruisseau l'avait pris \\
\hline 5 M. Oui, attends. \\
\hline 6 G. Il ne lui suffisait pas \\
\hline 7 G. Il prenait aussi ce beefsteak-là \\
\hline 8 M. Alors qu'est-ce qu'il fit? \\
\hline 9 G. Il alla dans l'eau et (10) le prit » \\
\hline
\end{tabular}

51 Analyse temporelle :

MQ(4) indéterminé AV MSiT(2) AV MSiT(6) AV MSiT(7) AV MSiT(9) AV MSiT (10)

Enoncés de la trame ${ }^{11}: 4,9,10$

Enoncés de l'arrière -plan11:2, 3, 6, 7

52 L'aspect est marqué en (2), (6) et (7) avec l'IMP, à fonction imperfective, pour les états mentaux du chien, et en (9) et (10) avec le PRe, à fonction perfective (aoristique). Les procès, téliques en (9) et (10) sont coordonnés à l'expression de la perfectivité, et duratifs en (2) et (6) à l'imperfectivité. A noter l'articulation correcte entre les trois formes (IMP, $\mathrm{PQP}, \mathrm{PRe}$ ) exprimant l'aspect, les relations temporelles, et les propriétés temporelles inhérentes des procès.

Dans une autre version, française, de cette histoire $(G .2 ; 11 ; 20)$ le déroulement de l'histoire est différent :

\begin{tabular}{|l|}
\hline Ex. 12 b) \\
\hline 1 G. Il voulait deux beefsteaks \\
\hline 2 P. Qu'est ce qu'il a fait avec deux beefsteaks? \\
\hline 3 G. Il a mangé un beefsteack et après... \\
\hline
\end{tabular}


4 il s'est jeté dans l'eau pour prendre l'autre beefsteak.

5 P. Et alors il n'a rien trouvé du tout ?...

6 Qu' est ce qui s'est passé....quand il a sauté dans l'eau...?

7 G. Que l'eau... ( réfléchit) elle a mangé le beefsteack

On a de (1) à (4) une succession de procès, et de (4) à (7) une ambiguïté : deux analyses sont possibles :

- MQ7 AV MSit4

- MSit4 CONT MSit7

Dans a), on doit inférer un PQP que G. aurait dû utiliser à la place du PC (individuation de trois points dans la ligne du temps). Dans b) le PC serait utilisé probablement à la place d'un IMP ou d'un PS (deux points temporels). L'analyse aspectuelle montre un contraste entre la fonction imperfective de (1) et la fonction perfective de (3), (4).

La simplicité morphologique de la version française par rapport à la précédente est à relier, nous semble-t-il, aux possibilités offertes par la langue française parlée aux enfants. L'absence du PS/PRe et l'alignement de la narration sur le PC atténuent les contrastes aspectuels. La versatilité du PC laisse à l'enfant la liberté entre plusieurs conclusions possibles, indéterminées.

57 Ces deux versions de la même histoire peuvent contribuer au débat sur les influences réciproques du développement cognitif et du développement linguistique : la possibilité pour l'enfant d'habitualiser précocement certaines capacités cognitives par l'intermédiaire des structures grammaticales et lexicales de la langue qu'il acquiert.

5 Les Temps italiens, très saillants, constitueraient pour cet enfant, selon nous, un schéma de base et un facteur d'ancrage des représentations mentales. A titre de validation de cette hypothèse on peut contraster le PRe + PQP italien vs le PC français des exemples précédents avec les SBJ IMP italien vs le SBJ PR français des ex. 13.

En 13) G. donne le même type de réponse pour justifier deux actions répréhensibles qu'il vient d'accomplir.

\begin{tabular}{|l|}
\hline Ex. 13 (G. $3 ; 0)$ (G. a démonté un jeu) \\
\hline a) 1.M. Perché hai staccato tutti i pezzi ? \\
\hline 2.G. Perchè non volevo che li mangiasse un cane \\
\hline « a) Pourquoi tu as détaché toutes les pièces \\
\hline Parce que je ne voulais pas qu'un chien les mangeât. » \\
\hline b) (à table) \\
\hline 1.P. Pourquoi tu as renversé ta soupe?
\end{tabular}


2.G. Parce que je ne veux pas que le chien la mange.

60 la subordonnée complétive. Le choix a pour effet de modaliser son explication d'excuse et de mettre l'accent sur son état mental AV MP. Le MQ est spécifié dans la question (1).

61 En français, il utilise le PR pour la principale et le PR SBJ (ici indistinct de l'Indicatif) pour la subordonnée. L'effet pragmatique est de persistance jusqu'au MP de l'état mental qui a déterminé l'action. La raison de la simplification morphologique de la formulation française est à rechercher dans l'absence du SBJ IMP dans l'input. En revanche, on observe un emploi massif du SBJ PR en français et italien à l'âge de $2 ; 9$, comme dans les énoncés suivants, tous syntaxiquement comparables :

\begin{tabular}{|l|}
\hline Ex. 14 (G. 2 ;10) \\
\hline a) Je veux faire rentrer Mimi ici pour qu'elle touche les prises \\
\hline b) Questo osserva gli animali che non si facciano male \\
\hline «b) Celui-ci observe les animaux qu'ils ne se fassent pas mal » \\
\hline c) Il faut taper l'eau pour qu'elle aille dans le trou \\
\hline d) Je pousse ça pour qu'il y ait de la place pour marcher
\end{tabular}

62 Cette série de subordonnées de but emploie les connecteurs pour que en français et che en italien, plus le SBJ dans les deux cas.

\begin{tabular}{|l|}
\hline $\begin{array}{l}\text { Ex. } 15 \text { (G. } 2 ; 10) \text { (Papa lui expliquait que sa bicyclette et celles de Papy et Mamy avaient été } \\
\text { volées) }\end{array}$ \\
\hline G. 1. Je vais prendre mon tricycle \\
\hline 2. et je vais le donner \\
\hline 3. pour acheter une bicyclette à Papy \\
\hline P. Mais tu es très gentil avec moi, G. \\
\hline G. 4. Je vais laisser ma bicyclette \\
\hline 5. et je vais partir en Italie, \\
\hline 6. et après je vais revenir \\
\hline 7. et après je vais reprendre ma bicyclette \\
\hline 8. et aller dans un autre village \\
\hline
\end{tabular}


9. et acheter une bicyclette pour Papa et une pour Papy.

L'ex 15 est intéressant par la quantité de procès considérés et par une division en deux parties, la première étant la présentation de la deuxième. (1) et (2) formulent l'intention d'action globale avec le but 3 . Après réaction favorable, il poursuit en segmentant et hiérarchisant la macro-action en micro-procès successifs : (4) à (9), en ordre chrono logique. La projection temporelle vers l'avant caractérisée par un emploi massif du FP et de et après est corrélée à une projection spatiale impliquant plusieurs déplacements. Une certaine cohésion est obtenue grâce aux verbes itératifs revenir et $\mathrm{r}$ eprendre qui distinguent clairement ces actions de celles indiquées auparavant, le voyage de retour opposé à l'aller, et à l'aide d'autre village qui fait référence à un autre lieu que celui où il est revenu. La complexité spatiale de ce récit témoigne d'un certain degré d'abstraction nécessaire à la conception d'un projet ; la faculté cognitive de réversibilité est également mise en jeu. Pour l'expression de la notion de proximité associée au futur, il peut être intéressant de comparer ce récit avec les exemples 16.

\begin{tabular}{|l|}
\hline Ex. 16 \\
\hline a)(G. 2 ;9) (à M. en indiquant la photo d'une jeune fille) \\
\hline Eva diventerà così \\
\hline a) « Eva deviendra comme ça » \\
\hline b) 1. Questa è Eva 2. quando sarà grande \\
\hline b) « Celle-ci est Eva quand elle sera grande » \\
\hline c) (G ; 2 ;10) (à table avec P. et Eva) \\
\hline P. Elle mange rien cette fille là \\
\hline \hline G. Mimi (=Eva) après, elle va mourir de faim \\
\hline
\end{tabular}

64 En français on trouve la forme périphrastique pour renvoyer à l'avenir proche. En italien, a) et b), le FUT exprime la notion de futur distant. En a) Eva est comparée à la fille de la photo à l'aide du verbe diventare $(+\mathrm{CH})$ et à la forme FUT. En b) il y a identification de deux personnages avec le présentatif (1) et une assimilation de la temporalité chronologique à la temporalité fictive (2). (2) introduit explicitement un MQ. On peut exclure ici une interprétation épistémique ${ }^{12} \mathrm{du}$ FUT puisque Eva est encore en devenir, et opter pour une interprétation temporelle.

A nouveau sur la notion de futur, les ex. 17 montrent des alternances de formulation avec les Temps PR (a) et FUT (b).

Ex. 17 
a) $($ G. $3 ; 0)$ (à la fin d'un jeu de construction d'une tour)

$1 \mathrm{M}$. Il primo che la butta in terra non mangia la caramella

(G. était en train de la jeter par terre)

2G. Eva la butta in terra (Eva ne l'a pas encore jetée)

3 G. (Eva l'a jetée) Eva non mangia la caramella perchè l'ha buttata in terra.

«1 M. Le premier qui la jette par terre ne mange pas le bonbon

2 G. Eva la jette par terre

3 G. Eva ne mange pas le bonbon parce qu'elle l'a jetée par terre »

b) (G.3) (M. invite G. à aller dîner)

$1 \mathrm{M}$. Vieni si va a mangiare la pappa

2 G. L'ha mangiata il lupo la pappa

3 M. No, perchè non l'ho ancora preparata

4 G. La mangerà (il lupo)

«1 M. Viens on va manger la soupe

2 G. L'a mangée le loup la soupe

$3 \mathrm{M}$. Non, parce que je ne l'ai pas encore préparée

4 G. Il la mangera "

En a), nous pouvons évaluer la compréhension de l'énoncé (1), qui présente des relations d'ordre, compliquées par une phrase négative opposée à une affirmative, et où les deux verbes au PR ont une fonction injonctive. (2) et (3) montrent une compréhension exacte de (1) : en (2), l'enfant utilise le PR comme la mère pour s'exclure soi-même de cette action. Donc il s'agit pour lui d'attendre les mouvements d'Eva. En (3), il relie les situations futures et passées par une relation causale, en contrastant une fonction future exprimée encore avec un PR à une passée (+ADJ) exprimée avec un PPx.

67 En (b) l'enfant utilise d'abord un PPx en (2) et corrige en (4) par un FUT motivé de manière écrasante par le feedback de la $\mathrm{M}$, invalidant sa première affirmation. La fonction déictique de postériorité du FUT indique certitude et volition. Au niveau sémantico-pragmatique l'emploi des verbes en (2) et (4) est comparable à celui de la langue adulte. Comme dans la séquence de l'ex. 5 Maintenant est demain, l'enfant pense pouvoir changer la réalité matérielle par un changement linguistique.

Dans le texte qui suit, G., désirant rapprocher le MSit, n'a pas hésité à changer la configuration temporelle MSit APR MP en MP APR MSit (avec un PC pour le MSit) 
69 Ex. 18 (G.3) (A table. G. parle avec P. On attend Alexis et sa famille. G. ne le connaît pas mais il est curieux de le rencontrer.)

\section{G. Maintenant il vient Alexis}

2. P. Non

3. G. Maintenant il vient Alexis. 4.Pas ce soir il vient Alexis

5. P. Pas ce soir. Il vient cet après midi.

6. G. Non, pas cet après midi...

7. P. Si ils viendront....

8. G.j'veux aller au parc avec Alexis

On va s'habiller?

9. P. Mais il n'est pas encore arrivé Alexis

...

10. P. Il va venir Alexis. Pour l'instant il n'est pas là

Il va sonner à la porte. Quand il sonnera on ira le chercher $\cdots$

11. G. tu m'amènes chez Alexis quand il a sonné à la porte

12. G. Maintenant il est venu sonner à la porte

13. Viens sonner Alexis! (ton impératif)

14. Viens sonner avec ta voiture, Alexis!

15. Viens sonner, ne casse pas ma maison Alexis, d'accord ?...

16. P. Il ne va pas la casser, t'inquiète pas

17. G. Ne casse pas ma brosse à dents !....Casse pas avec ta voiture! ...

18. G. Pa, je veux descendre (Rép)....

19. G. On va aller voir Alexis

20. P. Il n'est pas là encore. 
21. G. Il a sonné à la porte Alexis.

22. P. Tu l'as entendu?

23. G. Oui.

24. G. Pourquoi tu l'as pas entendu, Alexis ?

25. P. Moi je ne l'entends pas, moi. Miller (1976), Calleri (1990)), des apprenants adultes de français L2 (Noyau 1991, 1997) et italien L2 (Giacalone Ramat 1990, 1993a,b), des enfants bilingues franco-allemands (Schlyter 1990, Meisel 1985) montre une divergence considérable quant à la variété des formes productives, aux niveaux et aux rythmes de développement de la morphologie verbale. Les adultes apprenants d'italien L2 montrent une précocité d'émergence d'oppositions morphologiques par rapport aux apprenants du français L2 qui vont très lentement dans l'acquisition de la morphologie verbale. Noyau (1990) a souligné l'opacité de la morphologie verbale française :

«La marque multi-fonctionnelle du français oral $[-e]$ résiste à toute hypothèse simple de la part des apprenants et reste forme non-marquée. » (1990 : 232-33). Giacalone Ramat. En ce qui concerne le parcours développemental, les résultats de Meisel, Schlyter ${ }^{13}$, Calleri, malgré les écarts d'âge, peuvent être rapprochés des nôtres. 
Pour le débat aspect / temps, les résultats d'Antinucci \& Miller (1976) ${ }^{14}$ et de Giacalone Ramat (1993 a : 14), selon qui le développement de la marque du passé est régi par les catégories de l'actionalité et de l'aspect, contrastent avec les nôtres.

\subsection{Enfants slaves / Enfants italiens}

sur le plan conceptuel le déplacement, il conclut que les enfants italiens sont obligés d'utiliser l'aspect perfectif pour exprimer le passé parce qu'ils n'ont pas encore construit la distinction passé / non passé, dominée par la catégorie de l'aspect imperfectif ( $\mathrm{p}$. 85-86). fonction temporelle mais modale épistémique. Dans nos données (autour de deux ans), cet emploi modal est réalisé par le Temps PR, alors que l'IMP a une fonction temporelle et aspectuelle.

\section{Résultats}

A) Cet enfant peut être défini comme un bilingue équilibré : jusqu'à l'âge considéré les deux langues se sont développées de manière équivalente et presque en parallèle : si différence il y a elle est due plutôt aux caractéristiques intrinsèques des langues de l'input. La capacité à utiliser les équivalents des types des discours français ou italiens au niveau fonctionnel et pragmatique en faisant des extensions d'une langue à l'autre s'est révélée. 
83 B) La généralisation de presque tout le paradigme verbal en italien et en français dans le système TS : en quelques mois jusqu'à l'âge de $2 ; 4$ les formes verbales d'emploi courant ont été mises en place. Ces formes correspondent déjà aux fonctions de la langue, elles sont plurifonctionnelles.

Les perspectives et les relations temporelles ont été développées simultanément : très précocement cet enfant a utilisé plusieurs formes passées du même verbe à fonction aspectuelle différente. Nous réfutons donc la théorie du Temps Défectif et en particulier la thèse soutenue par Antinucci \& Miller.

On a noté le développement presque simultané de plusieurs formes et fonctions verbales dont la production massive se succède très rapidement à partir de deux ans. Dès l'âge de $2 ; 5$ : l'emploi massif des constructions hypotactiques et de la subordination ; la référence hors du hic et nunc par rapport à un point de repère distinct du MP ; la référence à des événements (-PROX) sur l'axe temporel en particulier avec l'emploi du PR ou PPx et du FUT italien; des exemples où les relations et les perspectives temporelles et les caractéristiques inhérentes des verbes ont été mis en contraste ; tous semblent parler en faveur d'une saisie des notions temporelles que l'on peut considérer comme caractéristiques du système du temps de Référence (restreint puis libre).

86 C) Avec les exemples présentés, nous avons décrit quelques passages qualitatifs de la construction du système conceptuel de la temporalité sous-jacents au développement des formes verbales et inférés grâce à ceux-ci.

Le niveau de différenciation linguistique atteint à un âge assez précoce rend possible une exploration pointue de l'organisation conceptuelle et des modifications évolutives. On a pu expérimenter l'intérêt représenté par une analyse de type qualitatif. Notamment la grille d'analyse conceptuelle de la temporalité utilisée (cf. § 2) s’est révélée un instrument précieux d'exploration du langage enfantin.

D) Cet enfant, même en situation bilingue, a pu franchir les étapes de développement de la morphologie temporelle et des fonctions temporelles en termes des relations TP-TS-TR à un rythme comparable à celui observé chez les enfants slaves dont l'étude réalisée par Weist de la construction de la temporalité montrait un franchissement précoce des quatre phases citées. Ce constat lui avait servi pour postuler l'hypothèse que les différences des âges-type relevés entre enfants de langues maternelles différentes étaient probablement dues aux caractéristiques spécifiques des langues particulières. Les ensembles d'études inter-linguistiques de Dahl (1985) sur la typologie de la temporalité, et de Slobin (1985-1987) sur l'acquisition enfantine des langues, vont dans le même sens.

\subsection{Discussion}

Les résultats peuvent être induits par une série de variables :

- I. les caractéristiques de la langue italienne ;

- II. les caractéristiques de la langue italienne et de la langue française prises ensemble ;

- III. la capacité d'un bilingue de manier précocément la plurifonction-nalité.

En contraste avec la plurisémicité des morphes français qui semblent retarder une utilisation différenciée de la morphologie, nous croyons que la forte saillance des Temps italiens a joué un rôle d'ancrage des représentations mentales, favorisant ainsi également l'acquisition des formes françaises à travers leur identification fonctionnelle. 
91 L'exposition à deux langues typologiquement assez proches a pu permettre, selon nous, un exercice constant de comparaison des données facilitant un feedback linguistique et cognitif de chaque langue sur l'autre.

La ressemblance de nos résultats avec ceux de Weist concernant les enfants polonais nous a conduite à nous interroger sur ce constat. Si le parcours acquisitionnel de cet enfant, pour ce qui est de la construction de la temporalité, était explicable seulement par les caractéristiques de transparence et de synthéticité de la langue italienne, nos données auraient dû être proches de celles tirées des monolingues italiens, et s'il était explicable par le bilinguisme, à celles de tout autre enfant bilingue. Nous avons émis l'hypothèse que les deux langues concernées ici, prises dans leur ensemble, forment un système sémiotique capable de stimuler un travail cognitif d'identification, de reconnaissance, de séparation, d'intersection, de focalisation, de combinaison, semblable à celui offert aux enfants polonais par leur langue : une langue qui sépare et peut combiner de façon ortho gonale la dérivation lexicale sur le verbe et le traitement du temps. Nous avons examiné : 1) la formation morphologique du Temps, 2) la sémantique verbale.

93 1) L'opacité morphologique du français oral fournit selon nous à cet enfant la possibilité de se concentrer sur une partie phonétiquement quasi invariable des verbes, facilitant l'acquisition du thème verbal. L'origine latine des deux langues fait que ce thème est souvent quasi commun. La transparence morphologique de la langue italienne d'autre part permet une focalisation sur la partie variable. Nous suggérons que chaque langue a une tâche spécifique, et que l'axe opacité - transparence joue sur l'acquisition des deux aspects fondamentaux du phénomène morphologique verbal, vu comme composition de deux éléments : le thème et la désinence.

La désinence sert, pour l'italien et le français, en se combinant aux verbes, à réaliser des Temps à fonction temporelle et aspectuelle, tandis que la sémantique inhérente du verbe de son côté est transformée à l'aide d'un préfixe ou d'un préfixe plus un suffixe.

2) Le phénomène de la préfixation a frappé notre sujet assez précocement. Remarquons ici la tendance à former de nouveaux verbes en ajoutant le préfixe/ri-/ pour l'italien et / re-/pour le français au verbe pour exprimer la réitération, ou bien le renforcement ou l'achèvement avec des verbes statifs ou d'activité. Rapidement, au cours du développement, le préfixe ri/re a été employé massivement et avec des surextensions idiosyncrasiques, créant des nouveaux verbes ${ }^{15}$ dans le répertoire de l'enfant.

Un autre préfixe très utilisé par notre sujet est/s-/ en italien et /dé-/ en français : la traduction d'un verbe d'une langue à l'autre a révélé aussi des surextensions, avec la création d'un nouveau verbe qui peut exister ou non dans la langue courante. Par ex. à partir du français déborder il a crée l'italien sbordare ( $s^{+}$bordo (nom) + are (suffixe de l'INF italien))

Dans la langue polonaise, le phènomène de la suffixation et de la préfixation est central : les verbes à valeur perfective sont formés à l'aide d'un préfixe ou d'un suffixe à partir d'un verbe imperfectif fonctionnant comme thème invariable.

Bertinetto a remarqué que dans les langues slaves la distinction entre lexèmes " perfectifs » et « imperfectifs » donne vie à des vrais couples de verbes qui s'opposent sur le plan sémantique, du type de ceux qui apparaissent sporadiquement dans les langues romanes comme "sedette/si sedette»(p.111). Bertinetto avance l'hypothèse que la dichotomie établie classiquement dans les systèmes verbaux slaves entre verbes « perfectifs » et « imperfectifs » se refère, malgré la terminologie employée, au champ de 
la sémantique inhérente plutôt qu'à celui de l'Aspect (p. 244) ${ }^{16}$. Dans la description de Bertinetto, les langues slaves déploient un système dense d'oppositions lexématiques. C'est dans ce domaine qu'il note une affinité subtile entre système slave et roman :

«... Anche nel sistema verbale romanzo (...) è contemplata la presenza di coppie di verbi gemelli, lessicograficamente identici ma di diversa Azione (...) verbi superficialmente coincidenti, ma tali da esibire una distribuzione complementare in rapporto a tutta una serie di parametri sintattici (...) predicati azionalmente ibridi » (p. 318).

Les cas hybrides dans le système verbal italien sont la règle plutôt que l'exception. Cette hypothèse stimulante peut nous aider à rechercher dans la sensibilité que notre sujet a montrée, en même temps qu'il commençait à apparier formes et fonctions, pour ces "verbes à action hybride» ou pour les constellations formées par les couples lexématiques italien et français, une piste pour rendre compte des analogies remarquées dans le développement de la temporalité chez les enfants slaves et chez le nôtre. Dans son va-et-vient d'une langue à l'autre, il a pu découvrir des caractéristiques intrinsèques aux deux langues et utiles pour la construction de son système conceptuel de la temporalité. Lorsqu'une idiosyncrasie était ressentie par l'enfant, elle était toujours verbalisée avec des questions pertinentes. En résumé, la capacité à décomposer le verbe en préfixe, thème et suffixe, facilitée par les caractéristiques de transparence / opacité des deux langues, associée à leur parenté, a dû permettre à cet enfant de découvrir l'existence des verbes décrits ci-dessus, et créés accidentellement par lui-même, et à en parler pour comprendre leur sens caché et pour en dévoiler les ambiguïtés. C'est ce que reflète l'ex. 7 parmi beaucoup d'autres.

3) Nous arrivons au troisième point qui nous semble crucial. Slobin (1985) a défini, d'après Karmiloff-Smith (1979), le «principe opératoire » d'unifonctionnalité: « If you discover that a linguistic form expresses two closely related but distinguishable Notions, use available means in your language to distinctly mark the two Notions » (p.1228).

La difficulté pour l'enfant d'organiser intra-linguistiquement un système plurifonctionnel avait été étudiée et mise en relief par Karmiloff-Smith ( ibid ): « ... language development involves passing gradually from a series of juxtaposed unifunctional markers and processing procedures, to the intralinguistic organization of plurifunctional systemes of options for modulating meaning»(p. 1227). Selon Karmiloff-Smith le concept de plurifonctionalité semble absent du point de vue du jeune enfant.

Or, un enfant bilingue par nécessité doit se confronter dès le début non seulement au fait qu'une notion peut être exprimée par deux formes, mais aussi à ce que les morphèmes d'une langue sont, par rapport à l'autre, plurifonctionnels. Les complexités inhérentes au bilinguisme - la double tâche cognitive, le problème de l'identification et de la séparation des deux codes, la gestion du bilinguisme - ont pu stimuler les principes opératoires de stockage, analyse, comparaison, subdivision, appariement, postulés par Slobin, dans le parcours de développement. La complexification des relations temporelles, attestée en l'espace de quelques mois chez notre sujet, pourrait être interprétée à la lumière des principes opératoires activés précocement grâce au bilinguisme et, par exemple, le stade cognitif dit de "la réversibilité » être stimulé par la pratique des alternances codiques comme des exercices «d'aller-retour» d'une langue à l'autre, qu'un bilingue est obligé d'opérer au quotidien. Sans vouloir affirmer que l'exposition à deux langues soit en toute circonstance une facilitation, nous pensons que dans certains cas, notamment selon le type des langues en jeu, comme on l'a vu ici, la pratique bilingue peut constituer un 
apport au travail laborieux de l'enfant dans la construction et la mise en place des relations cognitives comme celles du domaine temporel.

\section{BIBLIOGRAPHIE}

ANTINUCCI, F. \& MILLER, R. (1976) “How children talk about what happened", Journal of Child Language 3,167-189.

BERNINI, G. \& GIACALONE RAMAT, A. (eds) (1990) La Temporalità nell'acquisizione di lingue seconde, Milano,Franco Angeli.

BERTINETTO, P.M (1986) Tempo, apetto e azione del verbo italiano. Il sistema dell' indicativo, Firenze, Accademia della Crusca.

BHARDWAJ, M., DIETRICH, R. \& NOYAU, C. (eds) 1988 Second langage acquisition by adult immigrant, Final Report. Vol V Temporality, Strasbourg, European Science Foundation.

CALLERI, D. (1990) « L'acquisizione dell' imperfetto in bambini di madre lingua italiana », in Bernini \& Giacalone Ramat.

COMBETTES, B., FRANÇOIS, J., NOYAU, C. \& Vet, C. « Les Aspects dans le discours narratif », Verbum 4 (1993).

COMRIE, (1976) Aspect, Cambridge University Press.

CORDIER, F. (1994) Représentation cognitive et langage, Armand Colin.

DAHL, O. (1985) Tense and Aspect Systems, Oxford, Blackwell.

DIETRICH, R., KLEIN, W. \& NOYAU, C. (eds) (1995) The acquisition of temporality in a second language, Amsterdam, Benjamins.

FLETCHER, P. \& GARMAN, M. (eds.) (1986) Language acquisition, Cambridge, C.U.P.

FRENCH, L. A. (1986) “The language of events”, in: Nelson,K. (ed.), Event Knowledge, Hillsdale NJ, Erlbaum.

Giacaloneramat, A. (1990) « Presentazione del progetto di Pavia sull' acquisizione di lingue seconde - Lo sviluppo di strutture temporali », in: Bernini \& Giacalone Ramat.

GiacAlone RAMAT, A. (1993a), “Tense and Aspect in Learner Italian", Linguistica.

GIACALONE RAMAT, A. (1993b) «Sur quelques manifestations de la grammaticalisation dans

l'acquisition de l'italien comme deuxième langue ", AILE 2, 297-322.

KARMILOFF-SMITH, A. (1979) “Some fundamental aspects of language development after age 5", in: Fletcher, P. \& Garman, M.

KLEIN, W. \& STUTTERHEIM, C. (1989) "Referential movement in descriptive and narrative discourse", in Dietrich, R. \& C. Graumann (eds), Language processing in social context, Amsterdam, North Holland, 39-76.

KLEIN, W. (1988) “Frame of Analysis”, in : Bhardwaj,M., DietrichR. \& C. Noyau. 
KLEIN, W. (1994) Time in Language, London, Routledge.

MEISEL, J. M. (1985) « Les phases initiales du développement de notions temporelles, aspectuelles et de modes d'action ", Lingua 66, 321-474.

NOYAU, C. (1991) la Temporalité dans le discours et dans l'acquisition, Thèse d'habilitation, vol. I, Université Paris VIII.

NOYAU, C. (1990) « Le développement de la temporalité dans l'acquisition initiale d'une langue étrangère ", in : Bernini \& Giacalone.

NOYAU, C. (1997) : Processus de grammaticalisation dans l'acquisition de langues étrangères : la morphologie temporelle. In : C. Martinot, ed. L'acquisition de la syntaxe en langue maternelle et en langue étrangère, Besançon, Annales Littéraires de l'Université de Franche-Comté, 223-252.

ROMEO, S. (1997a), "La participation de l'alternance codique au développement : observation de deux enfants bilingues en interaction. Etude longitudinale." Colloque International Alternance des langues et apprentissage, E.N.S. Fontenay-St. Cloud (fév. 1997), à par. dans Cahiers du français contemporain.

ROMEO, S. (1997b) “La granularité comme expression des schémas cognitifs dans les récits et les descriptions des systèmes techniques d'un enfant bilingue". Communication à la Table-Ronde du GdR 113, La Baume-lès-Aix.

ROMEO, S. (1997c) "Stratégies discursives et cognitives dans l'interaction de deux enfants bilingues. Etude longitudinale”, Communication au 1st International Symposium on Bilingualism, Vigo, Espagne.

SCHLYTER, S. (1990) "The acquisition of tense and aspect", in: J. Meisel (ed.)Two first languages, Dordrecht, Foris.

SCHWARZE, C. (1990) «I tempi verbali dell' italiano come sistema funzionale, concettuale e formale », in: Bernini \& Giacalone Ramat.

SLOBIN, D.I. (1985-1987) The crosslinguistic study of language acquisition, Hillsdale, Erlbaum.

STEPHANY, U. (1986) “Modality”, in: Fletcher, P. \& M. Garman (eds).

VYGoTSKY L. S. (1934 / 1985) : Pensée et langage. Ed. Sociales-Messidor.

WEIST, R. M. (1986) “Tense and aspect”, in: Fletcher, P. \& M. Garman.

WEIST, R.M. (1989) “Time Concepts in Language and Thought: Filling the Piagetian Void from two to five years" in: Levin, I,Zakay, D. (eds), Time and Human Cognition, Amsterdam, Elsevier-North Holland.

\section{NOTES}

1. Conventions d'abréviation des Temps verbaux français et italiens : Imparfait / Imperfetto IMP ; Passato Remoto PRe ; Passato Prossimo PPx ; Passé simple PS ; Passé composé PC ; Plus que parfait/ Trapassato Prossimo PQP ; Présent / Presente PR ; Futur /Futuro FUT ; Futur proche FP ; Participe passé / Participio Passato PP, Subjonctif/ Congiutivo SBJ (PR ou IMP) ; Conditionnel/ Condizionale COND; Participe présent / Participio presente PPR; Gérondif / Gerundio GER; Infinitif /Infinito INF ; Imperatif /Imperativo IFT. Afin d'éviter une confusion entre les Temps Verbaux et la dimension du temps physique ou des notions temporelles, on indique les premiers en abréviation ou avec l'initiale en majuscule et les autres intégralement et en minuscule. On 
utilise le terme "procès" "pour désigner les différents types de situations sous l'angle de leur constitution temporelle" (Noyau 1991: 48). Le terme "Action" utilisé par certains auteurs cités est employé avec le sens de : "constitution temporelle inhérente du verbe".

2. Pour les développements successifs, cf. Romeo 1997 a,b,c.

3. La grille d'analyse de la temporalité du programme ESF que nous avons adoptée dans cette étude est présentée dans Bhardwaj, Dietrich and Noyau (1988), Dietrich, Klein \& Noyau (1995). Une forme remaniée du modèle est proposée dans Klein (1994).

4. On s'est appuyé sur la description du système temporel de l'italien par Schwarze (1990).

5. Toutes les remarques concernant le système temporel de l'italien sont empruntées à Bertinetto (1986), et synthétisées à l'aide de la grille d'analyse ESF.

6. Les implications théoriques liées à ces Temps sont discutées à la section 5.1.

7. La question de la quantité d'information encodée par les langues est soulevée dans Combettes, François, Noyau \& Vet (1993) p.18. "Y a-t-il, à travers la diversité des langues, systématisation, spécialisation, de certaines caractéristiques linguistiques ?" (p. 32).

8. Les parcours détaillés de ces développements sont décrits dans notre recherche longitudinale

9. Abréviations utilisées dans la transcription: G. = Gianni, P. = papa, M. = mamma, E. = Eva, petite soeur de G. (Les énoncés de G. en français s'adressent à P., ceux en italien s'adressent à M.)

10. Selon Vygotsky, dans l'inter-relation précoce action / langage, le discours suit les actions, est provoqué et dominé par l'action ; à un stade plus avancé, il se déplace progressivement vers le point de départ du procès jusqu'à précéder l'action. Entre mot et action émerge alors une nouvelle relation : le langage guide, détermine et domine le cours de l'action. Les exemples 2, 3, $4,8,9,11,14,15,17$ et 18 illustrent bien cette théorie.

11. Cf. Klein \& Stutterheim (1989) pour le modèle du "mouvement référentiel" dans les textes, qui fonde sur des contraintes référentielles la distinction entre énoncés de la trame et énoncés de l'arrière-plan.

12. Pour la méthode d'identification de la valeur temporelle dans le FUT italien, cf. Bertinetto.

13. Schlyter retient les phénomènes de grammaticalisation des morphèmes temporels et la capacité de référer à un moment lointain (-PROX) sur l'axe temporel comme indices du dépassement du système ET.

14. Leur argument célèbre est fondé sur l'observation que jusqu'à $2 ; 5$, les enfants marquent le PP d'un verbe transitif en l'accordant en nombre et en genre avec l'objet de ce verbe. Cet accord, qui n'existe pas dans l'italien standard, est pour Antinucci \& Miller le signe que le PP est traité comme un adjectif et qu'il sert à décrire l'état final d'un événement ou d'une action.

15. Ainsi, risaltare peut vouloir dire en italien sauter à nouveau, mais aussi ressortir. Une étude approfondie de ce phénomène est menée dans notre recherche longitudinale en cours.

16. Comparer avec Comrie (1976: 19, 88-90).

\section{RÉSUMÉS}

Cet article porte sur l'acquisition du système de la temporalité, vue comme appropriation graduelle des catégories conceptuelles sous-jacentes aux manifestations linguistiques et inférables grâce à celles-ci. La perspective est développementale ; les choix méthodologiques se fondent sur une approche conceptuelle de la description des structures temporelles. Le sujet est un garçon bilingue italien-français. Les résultats montrent : 1) un développement équilibré des 
deux langues ; 2) la généralisation de presque tout le paradigme verbal en italien et en français avant $2 ; 4$ avec des formes correspondant aux fonctions de la langue, i.e plurifonctionnelles ; 3) les perspectives et les relations temporelles ont été acquises simultanément : la théorie dite « du Temps Défectif » est réfutée ; 4) dès l'âge de $2 ; 5$ saisie des notions temporelles caractéristiques du système de la référence ; remise en discussion de l'âge critique de l'acquisition de la séquence temporelle; 5) l'intérêt représenté par une analyse de type qualitatif; 6 ) résultats qui se rapprochent du développement observé chez les enfants polonais plutôt que de celui des enfants italiens ou français. Les résultats ont amené à une comparaison typologique entre langues slaves et romanes prenant en compte la formation morphologique du Temps et la sémantique verbale. L'acquisition de deux L1, avec les difficultés acquisitionnelles inhérentes au phénomène du bilinguisme, est vue, dans le cas présenté, comme capable de: faire découvrir des caractéristiques intrinsèques aux deux langues et utiles pour la construction du système conceptuel de la temporalité ; aider l'enfant à comprendre la plurifonctionnalité des formes ; stimuler précocement les opérations cognitives de mémorisation, analyse, comparaison, subdivision, organisation interne. Le type des langues en jeu semble une variable utile.

The study bears on the acquisition of the temporal system, seen as a gradual appropriation of the conceptual categories underlying linguistic manifestations. Our analysis follows a developmental perspective and a conceptual approach. The subject is an Italian French bilingual child. The results show that : 1) this child is a balanced bilingual ; 2) the generalisation of almost the whole verbal paradigm takes place before $2 ; 4$ with plurifunctional forms; 3) the simultaneous acquisition of temporal relations and perspectives disconfirms the Defective Tense hypothesis; 4) since age $2 ; 5$ the grasping of temporal notions typical of the Reference system contradicts the theory of a late development of temporal sequence ; 5) there is need of a qualitative analysis ; 6) such results are closer to the development of Polish children than to Italian or French. A comparison between Slavic and Roman languages follows, taking into account the morphological formation of tense and verbal semantics. The acquisition of two first language is seen, here, as able to : let the child discover the internal characteristics of the two languages, which turns to be useful for the construction of the conceptual system of temporality; help the child understand the plurifunctionality of forms; early stimulate the cognitive operating principles of memorizing, analysis, comparison, subdivision, mapping. The type of languages involved seems to be a useful variable.

\section{AUTEUR}

\section{SILVIA ROMEO}

Université Paris X-Nanterre, Modèles linguistiques et Dynamiques des langues (EA 372), et Groupement de Recherche sur l'Acquisition des Langues (GdR 113 C.N.R.S.) 